\section{Sonnenschutz für Babys ab 0 Monaten}

\author{
Ab März 2017 stehen auf dem Markt drei \\ neue Produkte zum Lichtschutz zur \\ Verfügung, die speziell für die Haut von \\ Babys und Kindern entwickelt wurden, \\ darunter auch Fotoprotector ISDIN \\ Pediatrics Fusion Fluid Mineral Baby - \\ der einzige Sonnenschutz für Babys ab \\ 0 Monaten. \\ Mineral Baby kann sowohl für das \\ Gesicht als auch für den Körper ver- \\ wendet werden und bietet optimalen \\ Schutz vor indirekten Sonnenstrahlen. \\ Enthalten sind $100 \%$ mineralische Fil- \\ ter wie Zinkdioxid und Titandioxid, \\ die ideale Verträglichkeit und einen \\ effektiven Rundumschutz bieten. Die \\ Tatsachen, dass Mineral Baby hypo-
}

allergen sowie von Kinderärzten und Dermatologen getestet wurde, runden das Profil von diesem Newcomer ab. Auch Babys mit atopischer Haut können ab dem Frühling von dem neuen Fotoprotector profitieren.

Hinzu kommt noch Fotoprotector ISDIN Pediatrics Transparent Spray Wet Skin, das speziell für Kinder und Wassersportler entwickelt wurde und auch auf nasser Haut aufgetragen werden kann. Ergänzt wird das Portfolio für die kleinen Helden durch Fotoprotector ISDIN Pediatrics Gel Cream, das Feuchtigkeit spendet wie eine Creme und schnell einzieht wie ein Gel. Die gesamte Pediatrics-Reihe zeichnet sich

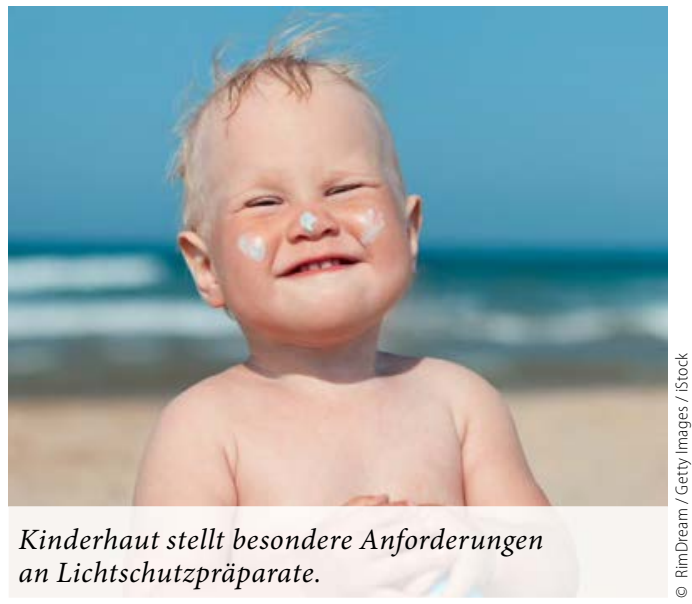

übrigens auch dadurch aus, dass sie rubbel-, extra wasserfest, biologisch abbaubar und speziell für die empfindliche Haut von Kindern formuliert wurde.

Nach Informationen von ISDIN

\title{
Mineralienpflege für gesunden Hautschutz
}

Feuchtigkeitsarme, trockene und empfindliche Haut kann sich häufig nicht selbst regenerieren und benötigt daher besondere Unterstützung. Die richtige Pflege kann helfen, die gestörte Hautbarriere wieder aufzubauen, den Fett- und Feuchtigkeitshaushalt der Haut zu verbessern und so vor weiterer Austrocknung zu schützen. Salthouse ${ }^{\circledast}$ hat eine therapeutische Gesichtspflege entwickelt, die speziell auf die Bedürfnisse feuchtigkeitsarmer, trockener und empfindlicher Haut abgestimmt ist.

Die Hydro-Aktivcreme ist reich an Hyaluronsäure und naturreinen TotesMeer-Mineralien, welche helfen, das interzelluläre Feuchthaltevermögen der Haut $\mathrm{zu}$ verbessern und langanhaltend mit Feuchtigkeit zu versorgen. Durch Panthenol und Vitamin E wird die Haut regene-

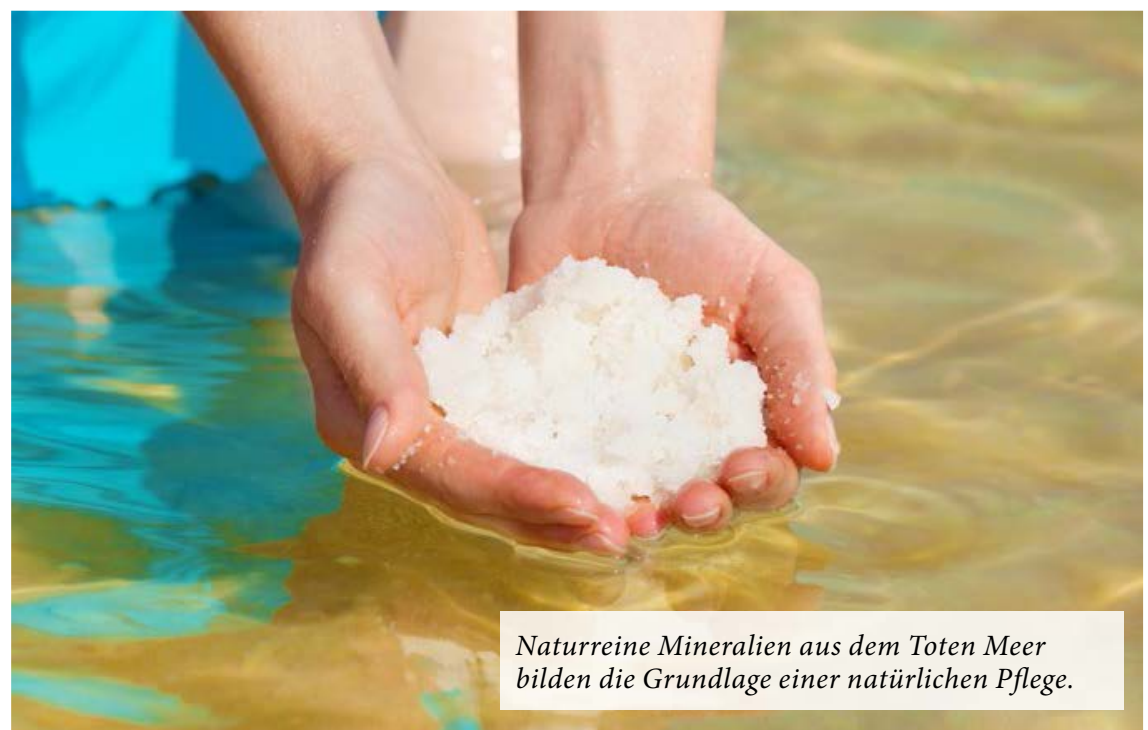

riert, die natürliche Hautschutzbarriere gestärkt und die Zellerneuerung gefördert.

Die Lipid-Aufbaucreme gleicht den Hydro-Lipidhaushalt der sehr trockenen Haut aus. Omega-Fettsäuren der Gruppe 3, 6 und 9 aus fünf wertvollen Naturölen (Distel, Sesam, Olive, Argan, Raps), wirken intensiv rückfettend und schützen die Haut vor dem Austrocknen. SheaButter, Vitamin E und Panthenol unterstützen die Zellregeneration, festigen die Hautstruktur und regeneriert sie mit hochwertigen Inhaltsstoffen.

Die Wasch-Emulsion ist die optimale Ergänzung für die extra milde Reinigung trockener und empfindlicher Gesichtshaut. Sie reinigt die Haut gründlich und besonders sanft. Gurkenextrakte sorgen für spürbare Erfrischung und Beruhigung leicht reizbarer Haut. Reich an essenziellen, ungesättigten Fettsäuren gleicht Nachtkerzenöl den Lipidmangel trockener Haut aus, verbessert den Zellstoffwechsel und schützt die Haut vor dem Austrocknen. Die Rezepturen sind frei von Parfum- und Farbstoffen, PEG, Silikon, Paraffin und Parabenen, dermatologisch getestet und $\mathrm{pH}$-hautneutral. Als Basispflege sind sie für Hauttypen mit Neurodermitis und Psoriasis geeignet.

Ingo Schroeder

Nach Informationen von The Salthouse, Egelsbach Veranstaltung: Salthouse ${ }^{\circledR}$ Presse-Forum, Köln, 10.11.2016 